\title{
Muséologies
}

Les cahiers d'études supérieures

muséologies

\section{L'exposition, un contexte de collaboration sur la scène mondiale de l'art : un cas de figure, l'art africain contemporain}

\section{Francine Couture}

Volume 9, numéro 1, 2018

Monde des Arts / Arts des mondes

URI : https://id.erudit.org/iderudit/1052626ar

DOI : https://doi.org/10.7202/1052626ar

Aller au sommaire du numéro

Éditeur(s)

Association Québécoise de Promotion des Recherches Étudiantes en

Muséologie (AQPREM)

ISSN

1718-5181 (imprimé)

1929-7815 (numérique)

Découvrir la revue

Citer cet article

Couture, F. (2018). L'exposition, un contexte de collaboration sur la scène mondiale de l'art : un cas de figure, l'art africain contemporain. Muséologies, 9(1), 33-43. https://doi.org/10.7202/1052626ar
Résumé de l'article

This analysis of the context of the globalization of the contemporary art scene is based on the concept of the cooperative network of the art worlds, as defined by the American sociologist Howard Becker, applied to the exhibition's sociological character. It is approached as a sociocultural event furthering the establishment of a cooperative network among artists, commissioners, critics and theoreticians who acknowledge in the exhibited works a certain number of values and ideas about art which they share to various degrees. Case studies from the corpus of contemporary African-art exhibitions that have been labelled as contemporary African art on the international stage serve as illustrations for this analysis.
Tous droits réservés (C Association Québécoise de Promotion des Recherches Étudiantes en Muséologie (AQPREM), 2018
Ce document est protégé par la loi sur le droit d'auteur. L'utilisation des services d'Érudit (y compris la reproduction) est assujettie à sa politique d'utilisation que vous pouvez consulter en ligne.

https://apropos.erudit.org/fr/usagers/politique-dutilisation/ 
Article un

L'exposition, un contexte de collaboration sur la scène mondiale de l'art: un cas de figure, l'art africain contemporain

Francine Couture 
Francine Couture a été professeure au département d'histoire de l'art de l'UQAM de 1974 à 2010. Elle est actuellement professeure associée à ce département. Ses travaux de recherche, guidés par la sociologie de l'art, interrogent les rapports de l'art contemporain avec l'institution artistique et le contexte social. Elle a dirigé la publication de deux livres portant sur les arts visuels au Québec dans les années soixante. Elle a également coordonné la publication des ouvrages collectifs suivants, Exposer l'art contemporain au Québec: Discours d'intention et d'accompagnement qui questionne le rôle de l'exposition dans la construction des identités culturelles, et Variations et pérennité des auvres contemporaines? portant sur la réexposition d'œuvres ayant un potentiel de variabilité. 
Ce numéro de la revue Muséologies propose une réflexion sur l'ouverture des disciplines de l'histoire de l'art et de la muséologie à l'étude de nouvelles aires géographiques et linguistiques et à de nouvelles formes artistiques qu'elles n'avaient pas traditionnellement considérées. J'ai retenu de la problématique de cette proposition la mention du concept de mondes de l'art du sociologue américain Howard Becker pour penser le contexte de la mondialisation de la scène de l'art contemporain et la notion d'exposition comme objet d'étude pertinent pour analyser des modalités de ses manifestations. L'exposition est un événement socioculturel rassemblant artistes, commissaires, critiques et théoriciens qui reconnaissent dans les œuvres exposées un certain nombre de valeurs et d'idées sur l'art qu'ils partagent à divers degrés. Elle favorise la constitution d'un réseau de coopération entre des acteurs qui, le plus souvent, prennent place dans un sous-segment du monde de l'art contemporain ${ }^{1}$. Je porterai mon attention sur ce caractère sociologique de l'exposition et l'appliquerai sur des études de cas prises dans le corpus d'expositions de l'art africain contemporain qui ont inséré le label ${ }^{2}$ art africain contemporain sur la scène internationale.

\section{Les analyses sociologiques de la mondialisation de l'art contemporain}

Rappelons que Becker conçoit les mondes de l'art ${ }^{3}$ comme des réseaux de coopération entre des individus concernés par une même discipline artistique. Cette coopération s'appuie sur le partage d'un ensemble de conventions qui constitue la culture artistique d'un groupe de professionnels œuvrant dans un monde de l'art, et qui sont aussi partagées par le public qui le fréquente. C'est en se référant à ces conventions que les individus de ce groupe entrent en interaction les uns avec les autres, et donnent

1 COUTURE, Francine. "L'exposition, une stratégie identitaire ". Sociologie de l'art. /1 (Opus 1\&2), 2003, p. 115-130.

2 La notion de label est empruntée à Raymonde Moulin. L'artiste, l'institution et le marché. Paris: Flammarion, 1992. 3 BECKER, Howard. Art Worlds. Berkeley, Los Angeles, London: University of California Press, 1982. de la signification à leurs actes et aux objets d'art produits et diffusés dans un secteur d'un monde de l'art.

La sociologie de l'art contemporain a étudié des réseaux de collaboration qui se sont développés dans le contexte de la mondialisation de la scène de l'art contemporain. Raymonde Moulin a démontré, qu'au cours des années 1980, l'internationalisation de l'art contemporain a résulté de l'action d'un nombre restreint de galeries leaders des grands centres artistiques des États-Unis et d'Europe, en étroite collaboration avec des méga collectionneurs, et que ce processus de valorisation artistique était indissociable de l'action des grandes manifestations internationales, telles les biennales et les grandes expositions dont les comités d'organisation ont contribué à la standardisation des choix des musées et des collectionneurs ${ }^{4}$. Elle a par ailleurs observé, qu'au cours des années 1990, on a assisté à une extension multiculturelle de l'offre artistique résultant de la multiplication des biennales et des foires et de la création de nouveaux musées, manifestations de la vitalité de nouveaux centres situés dans différentes régions du globe. Malgré cette ouverture, les réseaux de coopération mis en place dans les grands centres artistiques de l'Occident ont continué d'exercer leur autorité sur la définition des paramètres du label art contemporain. Les études du marché de l'art d'Alain Quemin ont confirmé cette analyse. Ce dernier a démontré que, bien que le rythme de circulation des ouvres et des personnes se déploie au-delà des frontières nationales, ces centres contrôlent la valorisation artistique et économique des œuvres sur le marché de l'art contemporain international. Il considère que ce marché est structuré par trois cercles : celui du noyau central formé par un duopole réunissant les États-Unis, et quelques pays européens dont principalement l'Angleterre et l'Allemagne, le cercle de la semi-périphérie occupé par des

4 MOULIN, Raymonde. L'artiste, l'institution et le marché. Paris: Flammarion, 1992 
pays qui ont une vie artistique se développant parallèlement à ce noyau central, ou qui peuvent éventuellement être en relation avec ce duopole, et la périphérie occupée par des pays qui ont établi peu de relations avec les centres artistiques du noyau central ou dont l'économie n'a pas atteint son développement. Cette analyse s'appuie sur l'étude des grandes foires d'art contemporain de Bâle et de Chicago ${ }^{5}$, ainsi que sur les listes du KunstKompass, d'Artfacts et celle d'ArtReview Power 100 classant les artistes au regard de leur reconnaissance par les galeries et musées leaders et par les comités des événements artistiques internationaux ${ }^{6}$.

Par ailleurs, sans remettre en question cette hiérarchie des nations dans le monde globalisé de l'art contemporain, la sociologie de l'art a aussi examiné d'autres modalités de coopération entre ses acteurs comme une des manifestations de sa globalisation. Des études se sont intéressées aux nouvelles connexions mises en place par des propriétaires de galeries de scènes artistiques dites périphériques avec le réseau de marchands d'art des grands centres artistiques. Ces analyses ont fait la démonstration de la structuration et de la vitalité de ces scènes ainsi que de la multiplication, depuis 10 ans, de leurs collaborations avec des professionnels des métropoles artistiques ${ }^{7}$.

\section{Le rôle de l'exposition sur la scène mondiale de l'art contemporain: un cas de figure, l'art africain contemporain}

Ce point de vue de l'analyse sociologique sur la mondialisation artistique peut guider l'étude d'expositions collectives affichant l'identité territoriale ou culturelle d'œuvres associées à la périphérie. Depuis 25 ans, sur la scène internationale, il s'est tenu un nombre impressionnant de ces expositions investies d'une signification identitaire. L'analyse de ces expositions permet de comprendre le rôle de médiation qu'elles ont joué dans la constitution de regroupements des professionnels du monde de l'art, d'étudier les modalités de leurs collaborations et de tracer la cartographie de leur déploiement géographique dans le monde de l'art.

Afin d'illustrer ce point de vue sociologique sur l'exposition, considérons l'exemple de l'entrée du label art africain contemporain sur la scène artistique mondiale en se référant à des récits qui relatent son émergence dans ce contexte de la mondialisation ${ }^{8}$. Ces récits mettent en valeur l'idée que cette émergence résulte du rôle joué par des expositions présentées sur la scène artistique internationale, en Europe et aux États-Unis, et non pas, dans une première étape, sur des scènes artistiques locales ou nationales. Leurs auteurs soulignent que ces expositions ont exercé une action déterminante dans la construction de réputations

(VELTHUIS Olav, BAIA CURIONI Stefano CURIONI dir.). Cosmopolitan Canvas, the globalization of markets for contemporary art. Oxford: Oxford University Press, 2015, p. 55-77. BRANDELLO, Armanda. "The Emergence of a Market in Brazil ". Dans VELTHUIS Olav et Stefano BAIA CURIONI (dir.). Cosmopolitan Canvas, the globalization of markets for contemporary art, Oxford: Oxford University Press, 2015, p. 215-237.

8 ENWEZOR,Okwui et Chika OKEKE-AGULU. Contemporary African Art since 1980. Londres: Damiani, 2009. AMSELLE, Jean-Loup. « Géopolitique de l'art contemporain africain ". Dans NDAYWEL é Nziem, Isidore, Elizabeth MUDIMBE-BOY (dir.). Images, mémoires et savoirs. Paris: Karthala, 2009, p. 195-200. CALMETTES, Delphine. Quel avenir pour l'art contemporain africain en Afrique après l'exposition Africa Remix. Master métiers et arts de l'exposition, Université de Rennes 2. Mémoire on line, < www.memoireonline.com>, consulté le 28 avril 2016. 
d'artistes d'origine africaine et la valorisation artistique de leurs œuvres. Certains d'entre eux ont aussi mentionné, qu'émanant de musées d'art ou d'organismes de diffusion du monde de l'art, ces expositions ont remis en question la conception exclusivement ethnographique de l'art africain pour en faire un objet du discours de l'esthétique9.

Les auteurs de ces récits s'entendent pour dire que l'exposition Les magiciens de la terre, présentée en 1989 à Paris, a contribué à sortir la catégorie de l'art africain contemporain de sa marginalité. On fait état des controverses qu'elle a soulevées à propos du choix des œuvres réalisé exclusivement par son commissaire Jean-Hubert Martin et ses collaborateurs ${ }^{10}$ et leur décontextualisation dans l'espace du Centre Pompidou et du Grand Hall de la Villette, ou encore à propos de la persistance de la notion de primitivisme comme trait spécifique de l'art africain contemporain ${ }^{11}$. Cependant, les auteurs de ces récits affirment que cette exposition est à l'origine d'un nouveau paradigme culturel ayant rendu compte de la diversité des lieux de la production artistique contemporaine dans le monde et remis ainsi en question la conception homogène et monolithique de la culture occidentale. On a alors assisté à un pluralisme géographique et esthétique rendant désormais possible l'entrée dans le monde de l'art contemporain à partir de différentes régions du globe.

Par ailleurs, les récits d'expositions relèvent qu'à partir des décennies des années 1990, et surtout 2000, le label art africain contemporain s'est imposé sur la scène artistique internationale. Par exemple, Okwui Enwezor et Chika Okeke-Agulu mentionnent l'action de l'exposition Seven Stories About Modern Art in Africa sur la réécriture de l'histoire de l'art africain dans une perspective mondiale ${ }^{12}$. Cette exposition présentée à la White Chapel à Londres en 1995 et coordonnée par Clementine Deliss avait réuni des commissaires d'origine africaine ${ }^{13}$ dont la sélection des œuvres rendait compte des échanges entre différents lieux de la vie artistique qui s'était développée en Afrique depuis les années 1960.

\section{Les récits de l'émergence de l'art africain} contemporain sur la scène internationale soulignent que ses expositions ont été propices à la formation et à la consolidation de réseaux de collaboration réunissant des artistes, des commissaires et des critiques d'art provenant d'une nouvelle génération d'origine ou de descendance africaine. Certains d'entre eux résidaient dans des villes africaines, mais un bon nombre faisait partie de la diaspora ${ }^{14}$ et œuvrait dans les milieux culturels européens et américains. Parmi eux, des commissaires d'exposition avaient acquis une reconnaissance dans le monde international de l'art contemporain, ce qui leur a donné accès aux ressources de ces grands organismes de diffusion, dont les biennales et les musées. Ces récits d'exposition mentionnent également le projet de ces professionnels de l'art de témoigner de l'identité des communautés africaines, de leur histoire marquée par l'esclavage, le colonialisme, le racisme ainsi que de leur inscription dans la modernité.

Leurs auteurs relèvent que les expositions ont été des contextes d'élaboration de nouveaux cadres théoriques pour penser la pluralité des manifestations des œuvres réunies sous le label art africain contemporain. Ils soulignent que ces discours critiques ont remis en cause l'autorité exercée sur la définition de l'art
9 ENWEZOR Okwui et al. Contemporary African Art since 1980. Londres: Damiani, 2009.

10 En Afrique, les commissaires des Magiciens de la Terre " ont jugé que les écoles copiaient les canons occidentaux et ils sont allés chercher des artistes subversifs ailleurs, dans des réseaux plus ruraux. Cette hétérogénéité a choqué. Les artistes africains ont eu le sentiment que l'on décidait pour eux, non pas avec eux ". COHEN, Annie, propos recueillis par Emmanuel Jardonnet. « Avec Les Magiciens de la Terre, on est passé d'un art mondial à un art global \%. Le Monde, 29 mars 2014, <www.lemonde.fr>, consulté le 29 avril 2016.
11 AMSELLE, Jean-Loup. " Géopolitique de l'art contemporain africain ". Dans NDAY WEL É NZIEM, Isidore et al. Image, mémoire et savoirs. Paris: Karthala, 2009, p. 196.

12 ENWEZOR Okwui et al., Contemporary African Art since 1980, Londres, Damiani, 2009.

13 Chika Okeke, Hadjii Sy, Salah M. Hassan, David Koloane, Wanjiku Nyachae.

14 Pour une réflexion théorique sur la notion de diaspora africaine: THOMPSON, Krista. «A Sidelong Glance: The Practice of African Diaspora Art History in The United States ". Art Journal, vol. 70, Issue 3, 2011, p. 6-31. 
contemporain par les centres artistiques occidentaux; ils abordent la question de l'authenticité de l'art africain au regard de ses divers paramètres identitaires, de son hybridité culturelle, son ancrage dans la mondialisation de la culture, le nomadisme des artistes et les modes d'inscription territoriale de leurs pratiques ${ }^{15}$.

Parmi les expositions souvent mentionnées figure Authentic/Ex-Centric: Africa In and Out of Africa présentée en 2001 à la Biennale de Venise dans le cadre du programme Africa in Venice $^{16}$. Les deux commissaires, Olu Oguibe, artiste et théoricien de l'art d'origine nigérienne professeur à l'University of Illinois, et Salah Hassan ${ }^{17}$ d'origine soudanaise et professeur à la Cornell University, étaient des représentants de la nouvelle génération de théoriciens et d'artistes engagés dans les études postcoloniale américaines. Ils étaient alors membres du groupe Forum for African Art qu'ils avaient créé en 2000 avec le théoricien et commissaire d'exposition Okwui Enwezor, et dont le mandat était de soutenir la participation de l'art africain aux grandes expositions internationales afin qu'il soit intégré au corpus de l'art contemporain ${ }^{18}$. Quatre ans auparavant, en 1997, Okwui Enwezor avait été le commissaire de la deuxième Biennale de Johannesburg. Et en 1994, avec Salah Hassan et Chika OkekeAgulu, il avait fondé la revue Nka Journal of Contemporary African Art vouée à la réflexion critique sur l'art produit par des artistes résidant en Afrique ou membres de sa diaspora dans le contexte de la mondialisation culturelle.

15 AMSELLE, Jean-Loup. « Géopolitique de l'art contemporain africain ». Dans NDAYWEL É NZIEM, Isidore et $a l$. Image, mémoire et savoirs. Paris: Karthala, 2009, p. 195-200. ENWEZOR, Okwuin et al, Contemporary African Art since 1980. Londres: Damiani, 2009.

16 Parmi les expositions présentées précédemment à la Biennale de Venise, on mentionne Fusion:West African Artists at the Venice Biennale (1993), qui réunissait cinq artistes provenant du Sénégal et de la Côte d'Ivoire. Son commissaire était Susan Vogel, qui était alors directrice du Museum for African Art à New York.

17 Salah Hassan a été l'un des commissaires de Seven Stories About Modern Art in Africa (1995).
Authentic/Ex-Centric: Africa In and Out of Africa réunissait sept artistes d'Afrique et de sa diaspora dont les installations, performances, vidéos et sculptures étaient exemplaires de pratiques artistiques conceptuelles dont les commissaires situaient l'origine sur les scènes africaines en 1970. En présentant ces œuvres à la Biennale, ces derniers ont voulu corriger la presque absence de l'art africain contemporain sur la scène internationale. La Biennale de Venise leur était apparue comme le lieu par excellence procurant aux exposants toute la synergie du monde de l'art contemporain nécessaire à leur reconnaissance. L'acte d'exposer donnait aux œuvres de la visibilité dans un espace public international et favorisait une éventuelle entrée dans l'histoire de l'art. Les œuvres choisies jetaient un regard critique sur les notions d'originalité et d'authenticité de l'art africain, déconstruisaient le point de vue colonial en abordant les thèmes de la transmission culturelle et des échanges entre les cultures africaines et européennes ${ }^{19}$. En les désignant comme des manifestations de l'art conceptuel africain, les commissaires accomplissaient une extension de l'utilisation de cette notion artistique qui, jusqu'à cette exposition, n'avait été appliquée qu'à des œuvres contemporaines occidentales.

La Documenta $X 1^{20}$, qui a eu lieu en 2002, est désignée par tous les récits d'exposition comme une autre étape importante de l'inclusion de l'art africain contemporain sur la scène internationale. Son commissaire était Okwui Enwezor, membre du Forum for African Art. Il avait conçu la Documenta comme une manifestation artistique et culturelle qui se déployait

18 PAULA, Iolanda. La Biennale de Dakar comme projet de coopération et de développement. Thèse de doctorat. École des Hautes Études en Sciences Sociales, Paris, 2011, p. 24.

19 OGUIBE, Olu et Salah HASSAN. " Authentic/ Ex-Centric at the Venice Biennal African Conceptualism in Global Context ", African Arts, vol. 34, n 4 (Hiver 2001), p. 64-75.

20 La Documenta de Kassel de 1992 est vue comme une exposition ayant ouvert la porte aux artistes africains. On y avait présenté, pour la première fois, deux artistes africains: l'artiste sénégalais Ousmane Sow et Mo Edoga d'origine nigérienne vivant en Allemagne. Les artistes africains ont été beaucoup plus présents à partir de la Documenta XI. 
sur la scène mondiale et mettait en cause la conception de sa structuration par les notions de centre et de périphérie. Il avait sollicité la collaboration de six commissaires engagés dans une réflexion sur les pratiques des artistes œuvrant en périphérie des métropoles artistiques. La Documenta XI était composée de plates-formes de réflexion qui avaient précédé l'exposition de Cassel et qui avaient eu lieu à Berlin, Vienne, Sainte-Lucie, New Delhi et Lagos. Ces dernières conjuguaient tablesrondes, conférences et projections de vidéos dont l'objectif était de " décrire le lieu actuel de la culture et ses interfaces avec d'autres systèmes de savoir mondiaux ${ }^{21}$ ". Ces platesformes, relève Delphine Calmettes, abordaient le contexte socioéconomique et politique des œuvres présentées provenant de divers lieux de la planète ${ }^{22}$. Okwui Enwezor avait déjà exploré ce modèle d'étalement géographique de la Documenta dans différentes régions du monde à la Biennale de Johannesburg en 1997, qu'il avait intitulée Trade Routes History and Geography. Il s'était entouré de six commissaires d'origines différentes pour interroger la place des espaces périphériques de création au sein d'une géographie mondiale de l'art ${ }^{23}$. Cette exposition, selon Jean-Loup Amselle, faisait explicitement référence au postcolonialisme, au multiculturalisme et à la globalisation ${ }^{24}$. La moitié des artistes invités étaient originaires de l'hémisphère sud. Par ailleurs, dans les conférences et les groupes de discussion ont été abordées les questions concernant la diaspora, l'exil, les conditions de vie d'artistes africains dans un grand centre artistique comme New York, et la répercussion de ces contextes sur leur production artistique et la représentation de leur identité.

Parmi les autres expositions marquantes d'art africain contemporain fréquemment mentionnées, notons Africa Remix, l'art contemporain d'un continent, présentée en 2005 au Centre Georges-Pompidou. Son titre affichait l'hybridité culturelle des œuvres exposées dans le but de communiquer les différentes conceptions des relations aux cultures africaines et occidentales élaborées par les auteurs de ces œuvres dans le contexte de la mondialisation. L'intention à son origine était donc de rendre compte de la complexité de l'identité de l'art africain « hybride et globale formée de différents apports des cultures d'origine et celle des pays d'intégration $^{25}$ " et de montrer la singularité et la diversité des pratiques artistiques réunies sous le label art africain contemporain ${ }^{26}$.

Son commissaire général était Simon Njami, co-fondateur de la Revue Noire, créée en 1991, et dont la création représentait " un événement majeur dans la fabrique de l'art contemporain africain ", parce qu'elle constituait " une réaction à l'exposition Les Magiciens de la Terre " ${ }^{27}$, jugée, par les fondateurs de la revue, encore attachée à la notion du primitivisme comme paramètre de l'art africain. Africa Remix réunissait environ 200 œuvres de 84 artistes:
21 Les thèmes des plate-formes ont été les suivants: «La démocratie, processus inachevé " à Berlin, «L'expérimentation de la vérité: les systèmes juridiques en évolution et les processus d'enquêtes et de conciliation " à New Delhi, "Créole et créolisation ” à Sainte-Lucie, « En état de siège: quatre villes africaines, Freetown, Johannesburg, Kinshasa et Lagos ", à Lagos. Documenta 11 Rétrospective, $<$ http://www.documenta.de/fr/retrospective/documenta11> consulté le 15 avril 2016.

22 Delphine Calmettes. Quel avenir pour l'art contemporain africain en Afrique après l'exposition Africa Remix. Master métiers et arts de l'exposition, Université de Rennes 2, 2008. Mémoire on line, <www.memoireonline.com>, consulté le 28 avril 2016

23 Id. "Quel avenir pour l'art contemporain africain en Afrique après l'exposition Africa Remix. Master métiers et arts de l'exposition, Université de Rennes 2, 2008, <www. memoireonline.com>, consulté le 28 avril 2016.
24 AMSELLE, Jean-Loup. « Géopolitique de l'art contemporain africain ". Dans NDAYWEL É NZIEM, Isidore et al. Image, mémoire et savoirs. Paris: Karthala, 2009, p. 196. 25 BASSENE, Reine. L'art contemporain africain: enjeux et perspective face à l'émergence d'un marché de l'art globalisé. Thèse de doctorat, Université de Nice, 2013, p. 71. 26 OUSSOU-ESSUI, Maeva. "Africa Remix, l'art contemporain d'un continent ", Histoire des expositions, Carnet de recherche du catalogue raisonné des expositions du Centre Pompidou. Centre Pompidou, <http://histoiredesexpos.hypotheses.org.>, consulté le 15 avril 2016.

27 AMSELLE, Jean-Loup, " Géopolitique de l'art contemporain africain ». Dans NDAYWEL É NZIEM, Isidore et al. Image, mémoire et savoirs, Paris: Karthala, 2009, p. 196. 
24 d'entre eux étaient issus de la diaspora et 62 résidaient en Afrique. Elle a été montrée à Düsseldorf, Stockholm, Londres, Tokyo et Johannesburg. Sa présentation sur le continent africain à la Johannesburg Art Gallery a été particulièrement soulignée par le directeur de cet organisme. Ce dernier a mentionné qu'elle avait ouvert la diffusion de l'art africain contemporain à l'extérieur des grands centres artistiques en étant sa première exposition majeure présentée en Afrique ${ }^{28}$.

La réalisation d'Africa Remix s'est appuyée sur la collaboration de quatre commissaires dont les activités professionnelles étaient bien ancrées dans le réseau mondial des expositions de l'art africain contemporain. Son commissaire général Simon Njami avait déjà réalisé des expositions d'art africain contemporain présentées en Europe. Et en 2004, il avait participé à la section africaine de la Biennale de Sao Paulo et avait également assumé la direction de trois " éditions " des Rencontres photographiques de Bamako ${ }^{29}$. Par ailleurs, Jean-Hubert Martin a été commissaire d'Africa Remix pour le Museum Kunst Palast, à Düsseldorf, dont il était le directeur: cette collaboration s'inscrivait dans la continuité de ses activités de commissaire des Magiciens de la Terre (1989) et de Partage d'exotismes (Biennale de Lyon, 2000). David Elliott avait organisé la première exposition d'art contemporain de l'Afrique du Sud au Museum of Modern Art (Oxford). En 2000, il avait été président du Comité international de la Biennale de Dakar, et en 2005, il était le directeur du Mori Museum (Tokyo) qui a reçu Africa Remix. La plupart des artistes exposés

28 KELLNER, Clive. "Preface ", Africa Remix contemporay, art of a continent. Johannesburg Art Gallery, p. 9, 2007, <https://books.google.ca>, consulté le 18 avril 2016.

29 CALMETTES, Delphine. Quel avenir pour l'art contemporain africain en Afrique après l'exposition Africa Remix.

Master métiers et arts de l'exposition, Université de Rennes 2, <www.memoireonline.com>, consulté le 28 avril 2016.

30 Ibid.

31 Présentation. Fondation Sindika Dokolo. <http://www. fondation-sindikadokolo.com/fr/presentation>, consulté le 28 avril 2016. avaient déjà participé à des expositions organisées par ces commissaires, dont Magiciens de la Terre et Partage d'exotismes ${ }^{30}$.

Certains d'entre eux ont aussi participé, en 2007, à l'exposition Check List Luanda Pop présentée à la $52^{\mathrm{e}}$ Biennale de Venise, pour laquelle a été construit le Pavillon africain. Notons d'ailleurs que, pour la première fois, des artistes africains étaient réunis dans un seul pavillon. Cette exposition regroupait 30 artistes issus de la collection de Sindika Dokolo, collectionneur africain d'art contemporain vivant à Luanda en Angola, et dont la fondation avait activement soutenu cette participation de l'art africain à la Biennale de Venise. Cette action rencontrait ses objectifs dont le développement de partenariats avec des institutions internationales et des coopérations avec d'autres villes et nations d'Afrique ${ }^{31}$. Les commissaires de Check List Luanda Pop étaient Simon Njami, qui avait été commissaire général d'Africa Remix, et Fernando Alvim ${ }^{32}$, artiste et vice-président de la fondation. Ce dernier avait été directeur de la Triennale de Luanda dont la première présentation en 2007 avait également été soutenue par la fondation Dokolo.

Simon Njami considérait que la présence de Check List Luanda Pop au Pavillon africain de la Biennale de Venise permettait « de poser un regard endogène sur les interactions, les échanges entre la diaspora et le continent, de faire le point sur ce qui se passe d'intéressant dans la création africaine ${ }^{33}$ ". Dans leur texte d'introduction à l'exposition, les deux commissaires ont souligné que Check List Luanda Pop constituait une nouvelle étape du récit des expositions de l'art africain contemporain

32 Fernando Alvim avait créé à Bruxelles un espace d'exposition Camouflage, "un satellite culturel africain en Europe ", relié au Center of Contemporary Art of Southern Africa.

Il souhaitait que la culture africaine colonise le monde, et avait le projet de créer une série d'antennes africaines fortes reliées entre elles. PENSA, op.cit. p. 58-60.

33 BOSO, Stéphane. "L'Afrique hisse pavillon à Venise ". Jeune Afrique.com. 11/08/07. Fondation Sindika Dokolo. <http://www.fondation-sindikadokolo.com/fr/lafrique-hissepavillon-a-venise-jeune-afrique>, consulté le 5 mai 2016. 
sur la scène internationale en rassemblant des ouvres de la collection de Sindika Dokolo, dont la fondation était un acteur majeur du monde de l'art africain ${ }^{34}$. Delphine Calmettes a relevé que les commissaires avaient donné à ce texte la portée politique d'un manifeste en défendant le pouvoir des Africains de définir leur propre destin. En effet, il se termine ainsi: «L'Afrique n'appartient à personne, nous sommes les premiers à l'affirmer. Mais il est cependant de la plus haute importance que son avenir soit défini, non pas à New York, Paris ou Londres, mais sur le continent même ${ }^{35}$ ".

\section{Les scènes artistiques africaines}

En 2007, en plus de soutenir la diffusion internationale d'œuvres contemporaines africaines, la fondation Sindika Dokolo a aussi joué un rôle important sur la scène artistique de Luanda en contribuant activement à la réalisation de la Triennale des arts. Son directeur était Fernando Alvim qui, lors de la première présentation de cet événement, a surtout exposé des œuvres de la collection Dokolo. Afin de renforcer le réseau international de la Triennale, on avait invité des acteurs de ce réseau à participer à des conférences et à des résidences d'artistes ${ }^{36}$. On a aussi retenu de cette première " édition » de la Triennale qu'elle avait été associée à un projet de réhabilitation de la ville par l'occupation de bâtiments, dont certains étaient inutilisés, et qui avaient été transformés pour le temps de l'exposition en lieux culturels ${ }^{37}$. De plus, on a souligné que de nombreuses activités de médiation destinées aux écoliers se sont tenues dans ces lieux.

La Triennale des arts à Luanda s'est ajoutée aux autres manifestations artistiques tenues en Afrique qui, depuis les années 1990, ont

34 Check list by Fernando Alvim et Simon Njami. Fondation Sindika Dokolo, <http://www.fondation-sindikadokolo.com>, consulté le 12 mai 2016.

35 Ibid.

36 PENSA, Iolanda. La Biennale de Dakar, projet de coopération et de développement, La Biennale de Dakar comme proje de coopération et de développement. Thèse de doctorat. École des Hautes Études en Sciences Sociales, Paris, 2011, p. 63. contribué à l'étalement géographique du monde de l'art contemporain. Mentionnons le rôle important de DAK'ART Biennale créée en 1990 par le gouvernement sénégalais et qui, dès l'« édition " de 1992, bien qu'elle se définissait comme panafricaine, a fait cohabiter les œuvres d'artistes africains et celles d'artistes de la scène internationale. À partir de 1996, la biennale a poursuivi cette stratégie d'internationalisation en sollicitant la collaboration de commissaires d'exposition des scènes européennes et nord-américaines ${ }^{38}$. Iolanda Pensa considère que DAK'ART Biennale a aussi permis aux artistes et aux commissaires travaillant dans divers pays africains d'exercer pleinement leurs pratiques et de consolider leurs réseaux de collaboration, et qu'elle a été ainsi une plateforme de réseautage entre ces acteurs du continent africain et les professionnels de la scène artistique internationale. Soulignons aussi les Rencontres africaines de la photographie de Bamako créées en 1994 afin de faire la promotion de la photographie africaine ainsi que le Salon Urbain de Doula (Sud), festival triennal d'art public organisé par le Centre d'art contemporain Douala, destiné aux pratiques urbaines et dont la première présentation a eu lieu en 2007.

\section{En conclusion}

Il faudrait analyser ces événements en profondeur pour bien comprendre leur rôle culturel sur les scènes artistiques nationales ou locales et les modalités de leurs relations avec les réseaux internationaux. Par ailleurs, ces cas de figure des expositions de l'art africain contemporain illustrent bien le rôle de médiation joué par l'exposition dans l'établissement de réseaux de coopération formés dans le but de faire la promotion d'un label artistique. L'examen de

37 Fernando Alvim avait créé un organisme SOSO qui s'était donné comme objectif de transformer les bâtiments abandonnés de la ville de Luanda en centres culturels afin de donner un nouveau souffle à la ville. PENSA, op.cit., p. 61 . 38 KONATE, Yacouba, La Biennale de Dakar, Pour une esthétique de la création africaine contemporaine - Tête à tête avec Adorno, Paris, Éditions Harmattan, 2009, p. 50-52. 
l'émergence de l'art africain contemporain sur la scène mondiale a démontré que ces expositions ont contribué à la constitution d'une communauté $^{39}$ transnationale regroupant des commissaires, des critiques, des théoriciens, des artistes résidant en Europe, aux États-Unis et en Afrique, réunis autour du projet de reconnaissance de l'art africain et ses discours critiques comme l'un des labels de l'art contemporain. Cette communauté s'est formée dans le contexte des grandes manifestations artistiques de la scène internationale. Elle est principalement associée à l'action de commissaires d'exposition issus de la diaspora africaine, travaillant en Europe et aux États-Unis, qui ont établi des collaborations avec les professionnels des institutions du noyau central du monde de l'art contemporain. Ce qui confirme les études menées par Raymonde Moulin et Alain Quemin sur le rôle joué par ces institutions sur l'inclusion d'un label artistique dans le palmarès international de l'art contemporain.

Retenons, par ailleurs, que les expositions d'art africain contemporain ont exercé une action déterminante sur l'extension de l'offre culturelle du monde de l'art en mettant en place des contextes d'énonciation de discours critiques qui ont remis en cause l'autorité culturelle des métropoles artistiques et leur conception exclusivement occidentale de l'histoire de l'art. Leurs commissaires ont également contribué à l'étalement géographique du monde de l'art contemporain en faisant circuler les expositions ou en organisant des événements sur des scènes artistiques dites périphériques au noyau central, ou encore, en collaborant à des manifestations artistiques organisées par des acteurs de mondes de l'art de villes africaines dans le but d'établir des contacts avec le monde international de l'art contemporain.

Afin d'évaluer la consolidation de l'inclusion de l'art africain contemporain sur la scène mondiale de l'art contemporain, il faudrait poursuivre cette analyse en examinant son degré d'insertion dans le marché de l'art et dans le réseau culturel des organismes de diffusion. Une évaluation du marché des œuvres africaines, datant de 2010, dit qu'entre 2002 et 2007, leurs prix ont été à la hausse, " portés par le boom des marchés émergents ${ }^{40}$ ", puis qu'ils ont chuté trois ans plus tard. Par ailleurs, la forte présence d'artistes d'origine ou de descendance africaine à la $56^{\mathrm{e}}$ Biennale de Venise (2015), sous la direction d'Okwui Enwezor, témoigne de la vitalité de leurs pratiques artistiques et de leur participation à la mondialisation artistique.
39 Pour la notion de communauté de goût, voir McEVILLEY, Thomas, " Ouverture du piège: l'exposition postmoderne et Magiciens de la Terre ", Les Magiciens de la Terre, Paris: Centre Georges-Pompidou, 1988, p. 20-23.
40 ARTPRICE, "L'art contemporain africain peine à vendre " (20 novembre 2013), <http//fr.artprice.com>, consulté le 31 mai 2016. 


\begin{abstract}
This analysis of the context of the globalization of the contemporary art scene is based on the concept of the cooperative network of the art worlds, as defined by the American sociologist Howard Becker, applied to the exhibition's sociological character. It is approached as a sociocultural event furthering the establishment of a cooperative network among artists, commissioners, critics and theoreticians who acknowledge in the exhibited works a certain number of values and ideas about art which they share to various degrees. Case studies from the corpus of contemporary African-art exhibitions that have been labelled as contemporary African art on the international stage serve as illustrations for this analysis.
\end{abstract}

